\title{
THE EFFECTS OF TEMPORARY CESSATION OF RENAL BLOOD FLOW IN RATS ${ }^{1}$
}

\author{
By SIMON KOLETSKY AND GORDON E. GUSTAFSON \\ (From the Institute of Pathology and Department of Surgery, Western Reserve University \\ School of Medicine, Cleveland, Ohio)
}

(Received for publication April 23, 1947)

Litten (1) observed in 1881 that temporary occlusion of the renal artery in dog and rabbit for $11 / 2$ to 2 hours was followed by tubular necrosis. In 1926 McEnery, Meyer and Ivy (2) clamped the blood vessels of both kidneys in dogs for periods of 30 minutes to 1 hour. This led to an increase in $\mathrm{BUN}^{2}$ and some animals died in uremia. Recently; Emmel (3) described the mitochondrial and $\mathrm{pH}$ changes in the rat's kidney following interruption and restoration of the renal circulation. Scarff and Keele (4) removed the right kidney of rabbits and then clamped the left .renal pedicle for periods up to 120 minutes. After release the BUN rose rapidly until death or reached a peak and declined with recovery. Outcome did not always depend on time of occlusion. Van Slyke et al (5) found that in the dog, if one kidney was removed and the renal artery of the other clamped for 3 hours, the animal would recover following an elevation of BUN. With clamping of 4 hours, only half of the dogs recovered and at 6 hours the procedure was uniformly fatal with uremic death in 4 to 8 days. Thus renal occlusion of more than 4 hours was considered irreversible. Selkurt (6) studied the effect of temporary clamping of the left renal artery of the dog on subsequent left renal clearances. Ischemia of 20 minutes' duration practically eliminated creatinine and paraamino-hippuric acid clearances during observations extending for 136 minutes after release of clamp.

In the following experiments a study was made of the effects of temporary complete renal ische$\mathrm{mia}$ in rats.

\section{METHODS}

Adult white male rats, weighing about 200 grams each, were anesthetized with intraperitoneal sodium pento-

1 Aided by a grant from the Elisabeth Severance Prentiss Foundation.

2 BUN denotes blood urea nitrogen. barbital given in dosage of 25 to $35 \mathrm{mgm}$. per $\mathrm{kgm}$. of body weight $(0.3$ to $0.4 \mathrm{ml}$. of a 2 per cent solution). With aseptic technique the kidneys were then exposed by lumbar incisions extending through the oblique abdominal muscles, the renal pedicles isolated, and the renal artery and vein occluded by means of bulldog clamps. The latter were applied on right and left sides usually within an interval of 20 seconds. Ureters were not occluded. Except for separation of adrenal from the kidney, no dissection of peri-renal fat was made. In all rats $0.5 \mathrm{ml}$. heparin was injected into the tail vein about 10 minutes before clamping to retard intrarenal thrombosis during the period of vascular occlusion. The heparin did not cause excessive bleeding. Food and water were withheld for about 10 hours prior to anesthesia, and were given ad libitum following the operation.

Complete renal ischemia was maintained bilaterally for exact periods of time, i.e., 30 minutes, 1 hour, 90 minutes, and 2 hours. The kidneys were generally dark purple at the end of these periods, but all became uniformly pink either within a matter of seconds or a few minutes after removal of the clamps. A group of 15 rats was studied at each time interval.

Renal function was followed by determination of BUN, made according to the method of Ormsby (7) with 0.1 or $0.2 \mathrm{ml}$. of blood from the tail. A control level was obtained prior to operation and then values were determined at fixed intervals following removal of the clamps, i.e., 6 hours, 24 hours, 2, 3, and 4 days, and in some instances 6 and 8 days. A sample of heart's blood was taken for BUN in rats which died in uremia.

The kidneys of all rats were removed for gross and microscopic study, either at the time of death or when the animals were sacrificed, usually from 4 to 8 days after the period of complete renal ischemia. A few rats in the 90-minute or 2-hour group showed renal infarction at autopsy and these were discarded and replaced. There was no observable post-operative infection.

Additional experiments indicated that the method used to produce complete renal ischemia was effective. In 5 rats coronal section of the kidneys after application of the clamps was not followed by bleeding from the cut surface. Profuse hemorrhage occurred when the clamps were released. Ten rats were sacrificed after periods of complete renal ischemia ranging from 30 minutes to 2 hours. In 2 of these animals 1 kidney was left unclamped to serve as a-control. Immediately after sacrifice, with the clamps still in place, the thoracic aorta was cannulated and perfused at an average pressure of $150 \mathrm{~mm}$. 


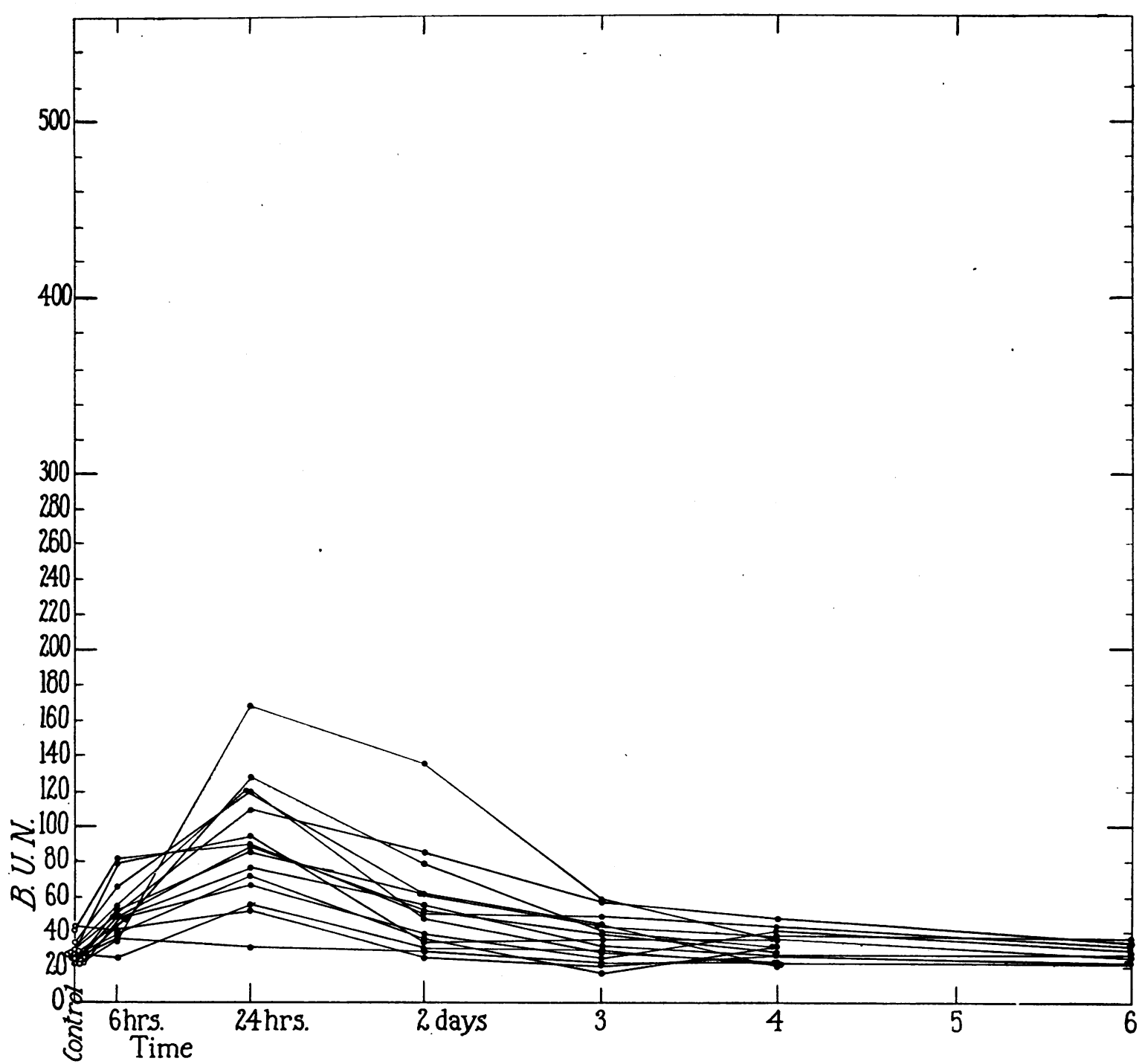

Fig. 1. Blood Urea Nitrogen following Complete Renal Ischemia for 30 Minutes

Hg with warm physiological saline followed by Higgin's india ink diluted 1 to 10 with saline. No gross coloration of the clamped kidneys was obtained. Microscopically, staining with india ink was practically negligible although pigment was observed occasionally in capillaries beneath pelvic mucosa and rarely in a few capillaries within the medulla.

\section{RESULTS}

After removal of the clamps, there was a progressive rise in BUN either until death or until a peak was reached, followed by a gradual decline and survival (Figures 1 to $4^{3}$ ). The ani-

${ }^{3}$ Values for BUN are in $\mathrm{mgm}$. per $100 \mathrm{ml}$. of blood. For convenience the time of death is given to the nearest day. mals which died showed typical manifestations of uremia, i.e., weakness, apathy, somnolence, and diarrhea. The mortality among the 4 groups of rats is recorded in Table $I$.

TABLE I

Mortality in 60 rats following complete renal ischemia

\begin{tabular}{c|c|c}
\hline \hline $\begin{array}{c}\text { Number of } \\
\text { rats }\end{array}$ & $\begin{array}{c}\text { Duration of complete } \\
\text { renal ischemia }\end{array}$ & Mortality \\
\hline & 30 minutes & per cent \\
15 & 1 hour & 0 \\
15 & 90 minutes & 13 \\
15 & 2 hours & 73 \\
15 & 100 \\
\hline
\end{tabular}


Fifteen rats with complete renal ischemia for 30 minutes. There was no mortality in this group. In 14 of the 15 rats the $B U N$ rose to a maximum 24 hours after return of renal circulation, the values ranging from 52 to 168 , with a mean of 94 . In 1 rat with a control BUN of 25 , a rise to only 37 was obtained at 6 hours. In every instance the BUN reverted to normal by the end of 4 or 6 days.

Fifteen rats with complete renal ischemia for 1 hour. Only 2 of the 15 rats in this group died, a mortality of 13 per cent. In the fatal cases death occurred 3 days after release of the clamps and the terminal BUN values were 312 and 400 , respectively.

Thirteen rats survived the renal ischemia. The maximum rise in BUN (105 to 256) usually occurred at 1 or 2 days. By the end of 4 or 6 days the values in 6 animals had declined to normal, but were still slightly elevated in the remaining 7, i.e., 40 to 82 .

Fifteen rats with complete renal ischemia for 90 minutes. Eleven of the rats died in uremia, a mortality of 73 per cent. Death occurred from 2 to 6 days after the renal circulation was re-

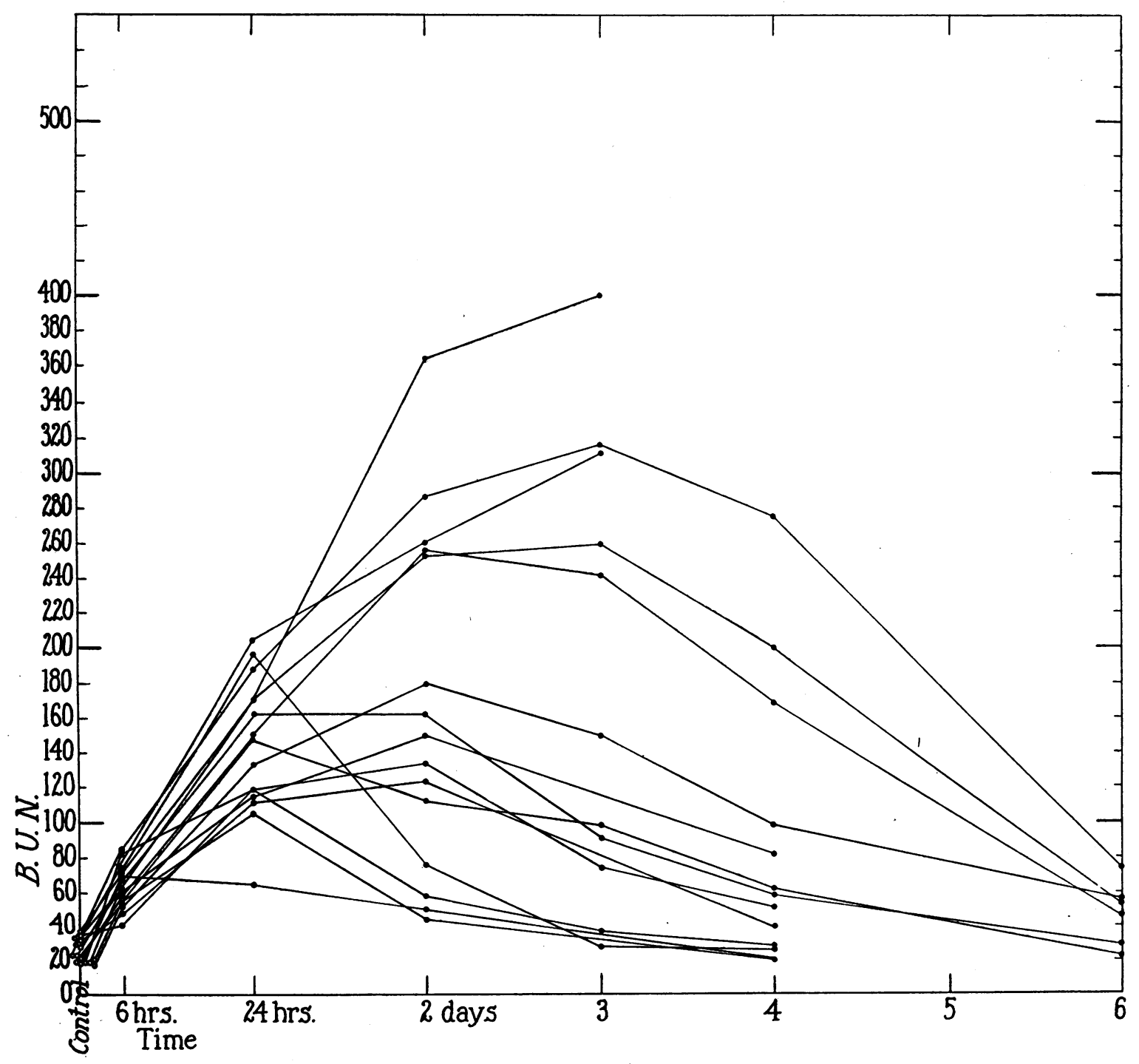

Fig. 2. Blood Urea Nitrogen following Complete Renal Ischemia for 1 Hour 
stored and the terminal BUN ranged from 244 to 560 .

Four animals in this group survived the renal ischemia after attaining a BUN ranging from 200 to 260 . At the time of sacrifice on the eighth day, the blood level was still slightly elevated in 3 rats (Figure 3 ).

Fifteen rats with complete renal ischemia for 2 hours. All rats in this group showed a progressive rise in BUN and died in uremia from 2 to 4 days after renewal of renal circulation (Figure 4).

\section{Morphology}

The kidneys of most rats in the 30 -minute group and of some in the 1-hour group showed no significant gross abnormality. In the remaining animals the kidneys were generally normal in size or slightly enlarged and frequently revealed bulging on section. The most distinctive change was the presence of a homogeneous cream-colored zone involving the entire inner portion of cortex. This contrasted with the outer cortex which was light red or brown. The medulla was pale pink and not remarkable.

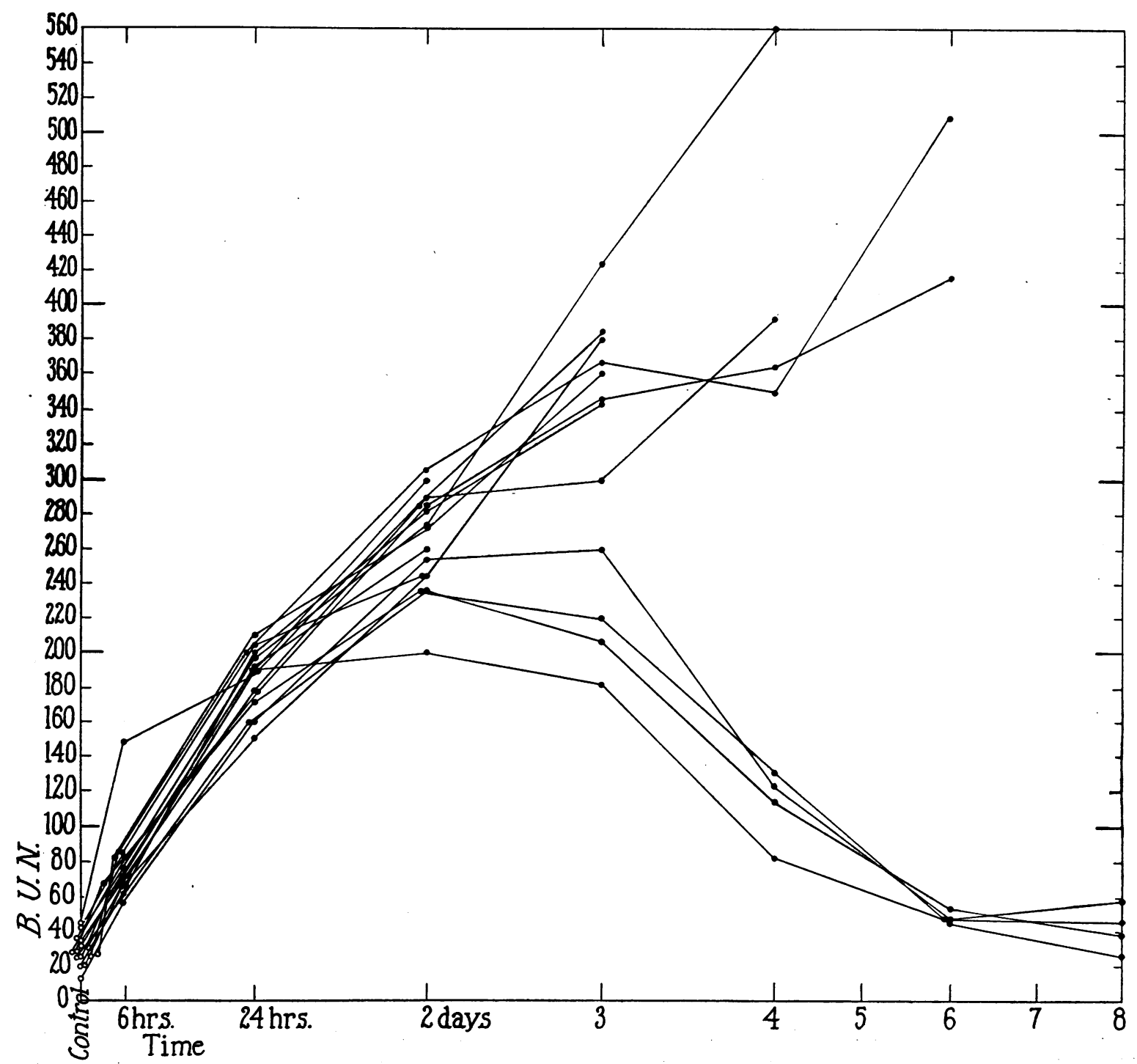

Fig. 3. Blood Urea Nitrogen following Complete Renal Ischemia for 90 Minutes 


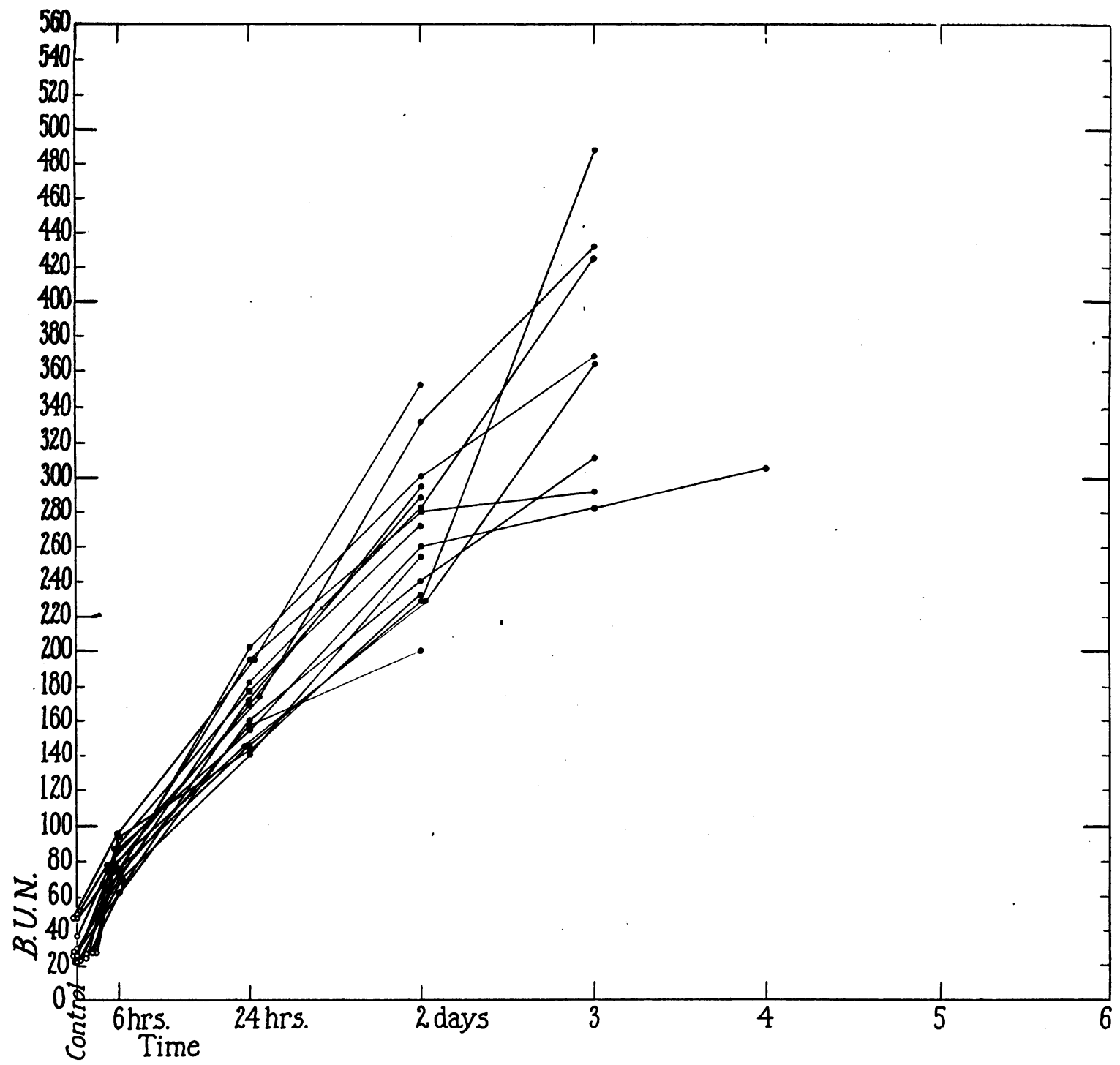

Fig. 4. Blood Urea Nitrogen following Complete Renal Ischemia for 2 Hours

Microscopically there was necrosis of tubules, involving principally proximal convoluted tubules in their distal or descending segments. The lesion was usually slight in rats with a 30-minute period of complete renal ischemia, more severe in degree and distribution in rats with ischemia of 1 hour's duration, and massive in animals which died in uremia, i.e., chiefly those in the 90-minute and 2-hour groups.

The necrotic tubules showed loss of nuclei and the lumens were occluded by coarse acidophilic débris derived from desquamated cells. Loops of Henle and distal convoluted tubules contained acidophilic hyaline casts, often with interspersed pyknotic nuclear fragments, but the epithelium was preserved. The lumens of the collecting tubules were filled with amorphous pink staining casts. In general the glomeruli showed no significant morphologic change although some were reduced in size. No lesion of blood vessels was found. In the vicinity of necrotic tubules there was a slight focal interstitial exudate of lymphocytes and a few polymorphonuclear leucocytes.

In the more severely injured kidneys, the desquamated material within the necrotic tubules was frequently the seat of superimposed calcific de- 
posit. This was negligible or absent in all rats with renal ischemia for 30 minutes and in most rats with ischemia of 1 hour's duration. The calcific material was in granular basophilic form in the early stage and later appeared as large, coarse, discrete or confluent masses attached to the lining of the tubules, often filling the lumens, and completely replacing the necrotic débris.

Regeneration of necrotic tubules was indicated by formation of new lining epithelial cells with vesicular nuclei and frequent mitoses. When sacrificed, all rats in the 30-minute group and most of those in the 1-hour group showed almost complete removal of necrotic material. The tubular lumens were generally dilated and lined by intact flat epithelium. There was marked reduction in the number of casts. Rats which died in uremia usually showed only slight or negligible repair. In 7 surviving rats, i.e., 4 in the 1-hour group and 3 with 90-minute renal ischemia, regeneration was incomplete since numerous tubules contained calcific masses in place of the necrotic débris. A similar change was also observed in several rats among the 90 -minute and 2-hour groups which died in uremia.

\section{DISCUSSION}

Rats readily survived a 30 -minute period of complete renal ischemia and usually survived a 1-hour period. The mortality was high (73 per cent) at 90 minutes and reached 100 per cent at 2 hours. Animals which died showed progressive elevation of BUN and death occurred in uremia from 2 to 6 days after renal blood flow was restored. In rats which survived, the BUN rose to a maximum from 6 hours to 3 days after release of the constricting clamps, depending on duration of the ischemia, and then returned to normal or slightly elevated values within the next few days.

Only 2 hours of complete renal ischemia were required to produce death consistently as compared to 4 hours or more in the dogs studied by Van Slyke et al (5). Presumably this is due to a difference in species. Difference in experimental procedure, i.e., simultaneous bilateral occlusion of renal vessels as against Van Slyke's technique of resecting one kidney and clamping the renal artery of the other, is evidently not significant. Following resection of the right kidney, we clamped the left renal artery and vein in 6 rats and the left renal artery alone in 6 additional rats. All 12 animals died in uremia from 1 to 3 days after a 2-hour period of vascular occlusion.

In our experiments, cessation of blood flow to the kidneys was produced by clamps which completely occluded main renal artery and vein. During the period of occlusion there was apparently no significant collateral circulation to the kidneys. Release of clamps was followed by almost immediate return of circulation and the subsequent presence of intact blood supply to the kidneys was confirmed morphologically by the absence of thrombosis or infarction. In all animals the renal lesion was uniform in both kidneys.

There was fairly good correlation between duration of renal ischemia and the resulting morphological injury to the kidneys after renewal of blood flow. With the longer periods of vascular occlusion, tubular necrosis of the kidneys was usually more diffuse and wider in distribution in the individual nephrons. Also, in rats which survived, the mean rise in BUN was more pronounced and sustained and was followed by a slower decline.

The principal change was necrosis of proximal convoluted tubules, especially the distal or descending segments. The reason for this localization is not known. The lumens of the tubules were blocked by coarse anuclear acidophilic masses, representing necrotic desquamated epithelial cells. Significant damage to epithelium of loops of Henle, to distal convoluted and collecting tubules, as well as to glomeruli, was not morphologically evident.

These observations are in contrast with the kidneys of human crush syndrome (8) or the experimental lesion in the rat (9), which show lower nephron nephrosis with involvement of the ascending loops of Henle and the distal convoluted tubules. The latter are often necrotic whereas the proximal tubules reveal only moderate degenerative change in the nature of cloudy swelling. The lesion also differs from that of rats with fatal tourniquet shock in which there is degenerative change, mainly of the proximal convoluted tubules, but no necrosis and only slight 
cast formation (10). However, because of the short survival time of such rats, from a few to several hours, the renal lesions are not strictly comparable with those due to complete ischemia.

In this study the damage to the kidneys was due primarily to the temporary period of complete anoxia during which the cells presumably underwent profound chemical alteration. However, return of circulation was required to bring about the development of cellular necrosis.

In rats with a 30-minute period of complete ischemia, the renal lesion was relatively slight and was followed by complete regeneration. This was also true of most rats in the 1-hour group even though kidney damage was more extensive. After 4 to 8 days the tubules were cleared of necrotic material and presented dilated lumens lined by newly formed flat epithelium. However, in 4 animals of the 1-hour group and in 3 surviving rats with 90 -minute renal ischemia, even though the majority of tubules were effectively restored, some revealed failure of regeneration associated with massive calcific deposit. Such tubular injury perhaps explains the failure of the BUN in these animals to return to normal at the time of sacrifice. Most rats which died in uremia showed only slight or negligible regeneration. This does not necessarily indicate an intrinsically irreversible lesion since death occurred before repair could be effective.

\section{SUMMARY}

1. Rats readily survived a 30 -minute period of complete renal ischemia and usually survived a 1-hour period. The mortality at 90 minutes was 73 per cent and at 2 hours the procedure was uniformly fatal.

2. After circulation was restored, the blood urea nitrogen rose rapidly until death, or reached a maximum level and then declined with survival of the animal.

3. The principal morphologic change was necrosis of the descending segments of the proximal convoluted tubules.

4. The renal lesion was not similar to that observed in human or experimental crush syndrome or in rats with fatal tourniquet shock.

\section{BIBLIOGRAPHY}

1. Litten, M., Ueber pathologische Verkalkungen und Kalkmetastasen in den Nieren. Virchows Arch., 1881, 83, 508.

2. McEnery, E. T., Meyer, J., and Ivy, A. C., Studies on nephritis; physiologic and anatomic changes following temporary ischemia of the kidneys. J. Lab. \& Clin. Med., 1927, 12, 349.

3. Emmel, V. M., Mitochondrial and $\mathrm{pH}$ changes in the rat's kidney following interruption and restoration of the renal circulation. Anat. Rec., 1940, 78, 361.

4. Scarff, R. W., and Keele, C. A., The effects of temporary occlusion of the renal circulation in the rabbit. Brit. J. Exper. Path., 1943, 24, 147.

5. Van Slyke, D. D., Phillips, R. A., Hamilton, P. B., Archibald, R. M., Dole, V. P., and Emerson, K., Effect of shock on the kidney. Tr. A. Am. Physicians, 1944, 58, 119.

6. Selkurt, E. E., The changes in renal clearance following complete ischemia of the kidney. Am. J. Physiol., 1945, 144, 395.

7. Ormsby, A. A., Direct colorimetric method for determination of urea in blood and urine. J. Biol. Chem., 1942., 146, 595.

8. Lucké, B., Lower nephron nephrosis. (The renal lesions of the crush syndrome, of burns, transfusions, and other conditions affecting the lower segments of the nephrons.) The Military Surgeon, 1946, 99, 371.

9. Corcoran, A. C., and Page, J. H., Post-traumatic renal injury; summary of experimental observations. Arch. Surg., 1945, 51, 93.

10. Unpublished observations of the authors. 